\title{
Coefficient Bounds for Certain Subclasses of $m$-Fold Symmetric Biunivalent Functions
}

\author{
Şahsene Altınkaya and Sibel Yalçın \\ Department of Mathematics, Faculty of Arts and Science, Uludag University, 16059 Bursa, Turkey \\ Correspondence should be addressed to Şahsene Altınkaya; sahsenealtinkaya@gmail.com
}

Received 27 July 2015; Accepted 17 November 2015

Academic Editor: Ming-Sheng Liu

Copyright (C) 2015 Ş. Altınkaya and S. Yalçın. This is an open access article distributed under the Creative Commons Attribution License, which permits unrestricted use, distribution, and reproduction in any medium, provided the original work is properly cited.

We consider two new subclasses $S_{\Sigma_{m}}(\alpha, \lambda)$ and $S_{\Sigma_{m}}(\beta, \lambda)$ of $\Sigma_{m}$ consisting of analytic and $m$-fold symmetric biunivalent functions in the open unit disk $U$. Furthermore, we establish bounds for the coefficients for these subclasses and several related classes are also considered and connections to earlier known results are made.

\section{Introduction}

Let $A$ denote the class of functions of the form

$$
f(z)=z+\sum_{n=2}^{\infty} a_{n} z^{n}
$$

which are analytic in the open unit $\operatorname{disk} U=\{z:|z|<1\}$, and let $S$ be the subclass of $A$ consisting of form (1) which is also univalent in $U$.

The Koebe one-quarter theorem [1] states that the image of $U$ under every function $f$ from $S$ contains a disk of radius $1 / 4$. Thus, every such univalent function has inverse $f^{-1}$ which satisfies

$$
\begin{aligned}
& f^{-1}(f(z))=z \quad(z \in U), \\
& f\left(f^{-1}(w)\right)=w \quad\left(|w|<r_{0}(f), r_{0}(f) \geq \frac{1}{4}\right),
\end{aligned}
$$

where

$$
\begin{aligned}
f^{-1}(w)= & w-a_{2} w^{2}+\left(2 a_{2}^{2}-a_{3}\right) w^{3} \\
& -\left(5 a_{2}^{3}-5 a_{2} a_{3}+a_{4}\right) w^{4}+\cdots .
\end{aligned}
$$

Function $f \in A$ is said to be biunivalent in $U$ if both $f$ and $f^{-1}$ are univalent in $U$. Let $\Sigma$ denote the class of biunivalent functions defined in unit $\operatorname{disk} U$.
For a brief history and interesting examples in class $\Sigma$, see [2]. Examples of functions in class $\Sigma$ are

$$
\begin{gathered}
\frac{z}{1-z}, \\
-\log (1-z), \\
\frac{1}{2} \log \left(\frac{1+z}{1-z}\right),
\end{gathered}
$$

and so on. However, the familiar Koebe function is not a member of $\Sigma$. Other common examples of functions in $S$ such as

$$
\begin{gathered}
z-\frac{z^{2}}{2}, \\
\frac{z}{1-z^{2}}
\end{gathered}
$$

are also not members of $\Sigma$ (see [2]).

For each function $f \in S$, function

$$
h(z)=\sqrt[m]{f\left(z^{m}\right)} \quad(z \in U, m \in \mathbb{N})
$$

is univalent and maps unit $\operatorname{disk} U$ into a region with $m$-fold symmetry. A function is said to be $m$-fold symmetric (see [3, 4]) if it has the following normalized form:

$$
f(z)=z+\sum_{k=1}^{\infty} a_{m k+1} z^{m k+1} \quad(z \in U, m \in \mathbb{N}) .
$$


We denote by $S_{m}$ the class of $m$-fold symmetric univalent functions in $U$, which are normalized by the series expansion (7). In fact, the functions in class $S$ are one-fold symmetric.

Analogous to the concept of $m$-fold symmetric univalent functions, we here introduced the concept of $m$-fold symmetric biunivalent functions. Each function $f \in \Sigma$ generates an $m$-fold symmetric biunivalent function for each integer $m \in \mathbb{N}$. The normalized form of $f$ is given as in (7) and the series expansion for $f^{-1}$, which has been recently proven by Srivastava et al. [5], is given as follows:

$$
\begin{aligned}
& g(w)=w-a_{m+1} w^{m+1}+\left[(m+1) a_{m+1}^{2}-a_{2 m+1}\right] \\
& \cdot w^{2 m+1}-\left[\frac{1}{2}(m+1)(3 m+2) a_{m+1}^{3}\right. \\
& \left.-(3 m+2) a_{m+1} a_{2 m+1}+a_{3 m+1}\right] w^{3 m+1}+\cdots,
\end{aligned}
$$

where $f^{-1}=g$. We denote by $\Sigma_{m}$ the class of $m$-fold symmetric biunivalent functions in $U$. For $m=1$, formula (8) coincides with formula (3) of class $\Sigma$. Some examples of $m$-fold symmetric biunivalent functions are given as follows:

$$
\begin{gathered}
\left(\frac{z^{m}}{1-z^{m}}\right)^{1 / m}, \\
{\left[-\log \left(1-z^{m}\right)\right]^{1 / m},} \\
{\left[\frac{1}{2} \log \left(\frac{1+z^{m}}{1-z^{m}}\right)^{1 / m}\right] .}
\end{gathered}
$$

Lewin [6] studied the class of biunivalent functions, obtaining the bound 1.51 for modulus of the second coefficient $\left|a_{2}\right|$. Subsequently, Brannan and Clunie [7] conjectured that $\left|a_{2}\right| \leq \sqrt{2}$ for $f \in \Sigma$. Later, Netanyahu [8] showed that $\max \left|a_{2}\right|=4 / 3$ if $f(z) \in \Sigma$. Brannan and Taha [9] introduced certain subclasses of biunivalent function class $\Sigma$ similar to the familiar subclasses. $S^{\star}(\beta)$ and $K(\beta)$ are of starlike and convex function of order $\beta(0 \leq \beta<1)$, respectively (see [8]). Classes $S_{\Sigma}^{\star}(\alpha)$ and $K_{\Sigma}(\alpha)$ of bistarlike functions of order $\alpha$ and biconvex functions of order $\alpha$, corresponding to function classes $S^{\star}(\alpha)$ and $K(\alpha)$, were also introduced analogously. For each of function classes $S_{\Sigma}^{\star}(\alpha)$ and $K_{\Sigma}(\alpha)$, they found nonsharp estimates on the initial coefficients. In fact, the aforecited work of Srivastava et al. [2] essentially revived the investigation of various subclasses of biunivalent function class $\Sigma$ in recent years. Recently, many authors investigated bounds for various subclasses of biunivalent functions (see $[2,10-15])$. Not much is known about the bounds on general coefficient $\left|a_{n}\right|$ for $n \geq 4$. In the literature, only few works determine general coefficient bounds $\left|a_{n}\right|$ for the analytic biunivalent functions (see [16-18]). The coefficient estimate problem for each of $\left|a_{n}\right|(n \in \mathbb{N} \backslash\{1,2\} ; \mathbb{N}=\{1,2,3, \ldots\})$ is still an open problem.

The aim of the this paper is to introduce two new subclasses of function class $\Sigma_{m}$ and derive estimates on initial coefficients $\left|a_{m+1}\right|$ and $\left|a_{2 m+1}\right|$ for functions in these new subclasses. We have to remember the following lemma here so as to derive our basic results.
Lemma 1 (see [4]). If $p(z)=1+p_{1} z+p_{2} z^{2}+p_{3} z^{3}+\cdots$ is an analytic function in $U$ with positive real part, then

$$
\begin{aligned}
\left|p_{n}\right| & \leq 2 \quad(n \in \mathbb{N}=\{1,2, \ldots\}), \\
\left|p_{2}-\frac{p_{1}^{2}}{2}\right| & \leq 2-\frac{\left|p_{1}\right|^{2}}{2}
\end{aligned}
$$

\section{Coefficient Bounds for Function Class $S_{\Sigma_{m}}(\alpha, \lambda)$}

Definition 2. A function $f \in \Sigma_{m}$ is said to be in class $S_{\Sigma_{m}}(\alpha, \lambda)$ if the following conditions are satisfied:

$$
\begin{aligned}
& \left|\arg \left(\frac{z f^{\prime}(z)}{(1-\lambda) f(z)+\lambda z f^{\prime}(z)}\right)\right|<\frac{\alpha \pi}{2} \\
& f \in \Sigma,(0<\alpha \leq 1,0 \leq \lambda<1, z \in U), \\
& \left|\arg \left(\frac{\lambda g^{\prime}(w)}{(1-\lambda) g(w)+\lambda w g^{\prime}(w)}\right)\right|<\frac{\alpha \pi}{2} \\
& (0<\alpha \leq 1,0 \leq \lambda<1, w \in U),
\end{aligned}
$$

where function $g=f^{-1}$.

Theorem 3. Let $f$ given by (7) be in class $S_{\Sigma_{m}}(\alpha, \lambda), 0<\alpha \leq 1$. Then,

$$
\begin{aligned}
& \left|a_{m+1}\right| \leq \frac{2 \alpha}{m(1-\lambda) \sqrt{\alpha+1}}, \\
& \left|a_{2 m+1}\right| \leq \frac{\alpha}{m(1-\lambda)}+\frac{2(m+1) \alpha^{2}}{m^{2}(1-\lambda)^{2}} .
\end{aligned}
$$

Proof. Let $f \in S_{\Sigma_{m}}(\alpha, \lambda)$. Then,

$$
\begin{gathered}
\frac{z f^{\prime}(z)}{(1-\lambda) f(z)+\lambda z f^{\prime}(z)}=[p(z)]^{\alpha}, \\
\frac{\lambda g^{\prime}(w)}{(1-\lambda) g(w)+\lambda w g^{\prime}(w)}=[q(w)]^{\alpha},
\end{gathered}
$$

where $g=f^{-1}$ and $p, q$ in $P$ have the following forms:

$$
\begin{aligned}
& p(z)=1+p_{m} z^{m}+p_{2 m} z^{2 m}+\cdots, \\
& q(w)=1+q_{m} w^{m}+q_{2 m} w^{2 m}+\cdots .
\end{aligned}
$$

Now, equating the coefficients in (13), we get

$$
\begin{aligned}
& m(1-\lambda) a_{m+1}=\alpha p_{m}, \\
& m(1-\lambda)\left[2 a_{2 m+1}-(\lambda m+1) a_{m+1}^{2}\right] \\
& \quad=\alpha p_{2 m}+\frac{\alpha(\alpha-1)}{2} p_{m}^{2}, \\
& -m(1-\lambda) a_{m+1}=\alpha q_{m}, \\
& m(1-\lambda)\left[(1+m(2-\lambda)) a_{m+1}^{2}-2 a_{2 m+1}\right] \\
& \quad=\alpha q_{2 m}+\frac{\alpha(\alpha-1)}{2} q_{m}^{2} .
\end{aligned}
$$


From (15) and (17), we obtain

$$
\begin{aligned}
p_{m} & =-q_{m}, \\
2 m^{2}(1-\lambda)^{2} a_{m+1}^{2} & =\alpha^{2}\left(p_{m}^{2}+q_{m}^{2}\right) .
\end{aligned}
$$

Also from (16), (18), and (20), we have

$$
\begin{aligned}
2 m^{2}(1-\lambda)^{2} a_{m+1}^{2}= & \alpha\left(p_{2 m}+q_{2 m}\right) \\
& +\frac{\alpha(\alpha-1)}{2}\left(p_{m}^{2}+q_{m}^{2}\right) \\
= & \alpha\left(p_{2 m}+q_{2 m}\right) \\
& +\frac{\alpha(\alpha-1)}{2} \frac{2 m^{2}(1-\lambda)^{2}}{\alpha^{2}} a_{m+1}^{2} .
\end{aligned}
$$

Therefore, we have

$$
a_{m+1}^{2}=\frac{\alpha^{2}\left(p_{2 m}+q_{2 m}\right)}{m^{2}(1-\lambda)^{2}(\alpha+1)} .
$$

Applying Lemma 1 for coefficients $p_{2 m}$ and $q_{2 m}$, we obtain

$$
\left|a_{m+1}\right| \leq \frac{2 \alpha}{m(1-\lambda) \sqrt{\alpha+1}} .
$$

Next, in order to find the bound on $\left|a_{2 m+1}\right|$, by subtracting (18) from (16), we obtain

$$
\begin{gathered}
4 m(1-\lambda) a_{2 m+1}-2 m(m+1)(1-\lambda) a_{m+1}^{2} \\
=\alpha\left(p_{2 m}-q_{2 m}\right)+\frac{\alpha(\alpha-1)}{2}\left(p_{m}^{2}-q_{m}^{2}\right) .
\end{gathered}
$$

Then, in view of (19) and (20) and applying Lemma 1 for coefficients $p_{2 m}, p_{m}$ and $q_{2 m}, q_{m}$, we have

$$
\left|a_{2 m+1}\right| \leq \frac{\alpha}{m(1-\lambda)}+\frac{2(m+1) \alpha^{2}}{m^{2}(1-\lambda)^{2}} .
$$

This completes the proof of Theorem 3.

\section{Coefficient Bounds for Function}

$$
\text { Class } S_{\Sigma_{m}}(\beta, \lambda)
$$

Definition 4. Function $f \in \Sigma_{m}$ given by (7) is said to be in class $S_{\Sigma_{m}}(\beta, \lambda)$ if the following conditions are satisfied:

$$
\begin{aligned}
& \operatorname{Re}\left(\frac{z f^{\prime}(z)}{(1-\lambda) f(z)+\lambda z f^{\prime}(z)}\right)>\beta \\
& f \in \Sigma,(0 \leq \beta<1,0 \leq \lambda<1, z \in U), \\
& \operatorname{Re}\left(\frac{\lambda g^{\prime}(w)}{(1-\lambda) g(w)+\lambda w g^{\prime}(w)}\right)>\beta \\
& \quad(0 \leq \beta<1,0 \leq \lambda<1, w \in U),
\end{aligned}
$$

where function $g=f^{-1}$.
Theorem 5. Let $f$ given by (7) be in class $S_{\Sigma_{m}}(\beta, \lambda), 0 \leq \beta<1$. Then,

$$
\begin{aligned}
\left|a_{m+1}\right| & \leq \frac{\sqrt{2(1-\beta)}}{m(1-\lambda)} \\
\left|a_{2 m+1}\right| & \leq \frac{2(m+1)(1-\beta)^{2}}{m^{2}(1-\lambda)^{2}}+\frac{1-\beta}{m(1-\lambda)} .
\end{aligned}
$$

Proof. Let $f \in S_{\Sigma_{m}}(\beta, \lambda)$. Then,

$$
\begin{gathered}
\frac{z f^{\prime}(z)}{(1-\lambda) f(z)+\lambda z f^{\prime}(z)}=\beta+(1-\beta) p(z), \\
\frac{\lambda g^{\prime}(w)}{(1-\lambda) g(w)+\lambda w g^{\prime}(w)}=\beta+(1-\beta) q(w),
\end{gathered}
$$

where $p, q \in P$ and $g=f^{-1}$.

It follows from (28) that

$$
\begin{aligned}
& m(1-\lambda) a_{m+1}=(1-\beta) p_{m} \\
& m(1-\lambda)\left[2 a_{2 m+1}-(\lambda m+1) a_{m+1}^{2}\right]=(1-\beta) p_{2 m} \\
& -m(1-\lambda) a_{m+1}=(1-\beta) q_{m}, \\
& m(1-\lambda)\left[(1+m(2-\lambda)) a_{m+1}^{2}-2 a_{2 m+1}\right] \\
& \quad=(1-\beta) q_{2 m} .
\end{aligned}
$$

From (29) and (31), we obtain

$$
\begin{aligned}
p_{m} & =-q_{m}, \\
2 m^{2}(1-\lambda)^{2} a_{m+1}^{2} & =(1-\beta)^{2}\left(p_{m}^{2}+q_{m}^{2}\right) .
\end{aligned}
$$

Adding (30) and (32), we have

$$
2 m^{2}(1-\lambda)^{2} a_{m+1}^{2}=(1-\beta)\left(p_{2 m}+q_{2 m}\right) .
$$

Therefore, we obtain

$$
a_{m+1}^{2}=\frac{(1-\beta)\left(p_{2 m}+q_{2 m}\right)}{2 m^{2}(1-\lambda)^{2}} .
$$

Applying Lemma 1 for coefficients $p_{2 m}$ and $q_{2 m}$, we obtain

$$
\left|a_{m+1}\right| \leq \frac{\sqrt{2(1-\beta)}}{m(1-\lambda)} .
$$

Next, in order to find the bound on $\left|a_{2 m+1}\right|$, by subtracting (32) from (30), we obtain

$$
\begin{aligned}
4 m & (1-\lambda) a_{2 m+1}-2 m(m+1)(1-\lambda) a_{m+1}^{2} \\
& =(1-\beta)\left(p_{2 m}-q_{2 m}\right) .
\end{aligned}
$$

Then, in view of (33), applying Lemma 1 for coefficients $p_{2 m}, p_{m}$ and $q_{2 m}, q_{m}$, we have

$$
\left|a_{2 m+1}\right| \leq \frac{2(m+1)(1-\beta)^{2}}{m^{2}(1-\lambda)^{2}}+\frac{1-\beta}{m(1-\lambda)} .
$$

This completes the proof of Theorem 5 . 
If we set $\lambda=0$ in Theorems 3 and 5 , then classes $S_{\Sigma_{m}}(\alpha, \lambda)$ and $S_{\Sigma_{m}}(\beta, \lambda)$ reduce to classes $S_{\Sigma_{m}}^{\alpha}$ and $S_{\Sigma_{m}}^{\beta}$ and thus we obtain the following corollaries.

Corollary 6. Let $f$ given by (7) be in class $S_{\Sigma_{m}}^{\alpha}(0<\alpha \leq 1)$. Then,

$$
\begin{aligned}
& \left|a_{m+1}\right| \leq \frac{2 \alpha}{m \sqrt{\alpha+1}}, \\
& \left|a_{2 m+1}\right| \leq \frac{\alpha}{m}+\frac{2(m+1) \alpha^{2}}{m^{2}} .
\end{aligned}
$$

Corollary 7. Let $f$ given by (7) be in class $S_{\Sigma_{m}}^{\beta}(0 \leq \beta<1)$. Then,

$$
\begin{aligned}
\left|a_{m+1}\right| \leq \frac{\sqrt{2(1-\beta)}}{m}, \\
\left|a_{2 m+1}\right| \leq \frac{2(m+1)(1-\beta)^{2}}{m^{2}}+\frac{1-\beta}{m} .
\end{aligned}
$$

Classes $S_{\Sigma_{m}}^{\alpha}$ and $S_{\Sigma_{m}}^{\beta}$ are, respectively, defined as follows.

Definition 8. Function $f \in \Sigma_{m}$ given by (7) is said to be in class $S_{\Sigma_{m}}^{\alpha}$ if the following conditions are satisfied:

$$
\begin{aligned}
& \left|\arg \left(\frac{z f^{\prime}(z)}{f(z)}\right)\right|<\frac{\alpha \pi}{2} \quad f \in \Sigma,(0<\alpha \leq 1, z \in U), \\
& \left|\arg \left(\frac{\lambda g^{\prime}(w)}{g(w)}\right)\right|<\frac{\alpha \pi}{2} \quad(0<\alpha \leq 1, w \in U),
\end{aligned}
$$

where function $g=f^{-1}$.

Definition 9. Function $f \in \Sigma_{m}$ given by (7) is said to be in class $S_{\Sigma_{m}}^{\beta}$ if the following conditions are satisfied:

$$
\begin{aligned}
& \operatorname{Re}\left(\frac{z f^{\prime}(z)}{f(z)}\right)>\beta \quad f \in \Sigma,(0 \leq \beta<1, z \in U), \\
& \operatorname{Re}\left(\frac{\lambda g^{\prime}(w)}{g(w)}\right)>\beta \quad(0 \leq \beta<1, w \in U),
\end{aligned}
$$

where function $g=f^{-1}$.

For one-fold symmetric biunivalent functions and $\lambda=0$, Theorems 3 and 5 reduce to Corollaries 10 and 11, respectively, which were proven earlier by Murugusundaramoorty et al. [19].

Corollary 10. Let $f$ given by (7) be in class $S_{\Sigma}^{\star}(\alpha)(0<\alpha \leq 1)$. Then,

$$
\begin{aligned}
& \left|a_{2}\right| \leq \frac{2 \alpha}{\sqrt{\alpha+1}} \\
& \left|a_{3}\right| \leq 4 \alpha^{2}+\alpha .
\end{aligned}
$$

Corollary 11. Let $f$ given by $(7)$ be in class $S_{\Sigma}^{\star}(\beta)(0 \leq \beta<1)$. Then,

$$
\begin{aligned}
& \left|a_{2}\right| \leq \sqrt{2(1-\beta)}, \\
& \left|a_{3}\right| \leq 4(1-\beta)^{2}+(1-\beta) .
\end{aligned}
$$

\section{Conflict of Interests}

The authors declare that there is no conflict of interests regarding the publication of this paper.

\section{References}

[1] P. L. Duren, Univalent Functions, Grundlehren der Mathematischen Wissenschaften, Springer, New York, NY, USA, 1983.

[2] H. M. Srivastava, A. K. Mishra, and P. Gochhayat, "Certain subclasses of analytic and bi-univalent functions," Applied Mathematics Letters, vol. 23, no. 10, pp. 1188-1192, 2010.

[3] W. Koepf, "Coefficients of symmetric functions of bounded boundary rotation," Proceedings of the American Mathematical Society, vol. 105, no. 2, pp. 324-329, 1989.

[4] C. Pommerenke, Univalent Functions, Vandenhoeck \& Ruprecht, Göttingen, Germany, 1975.

[5] H. M. Srivastava, S. Sivasubramanian, and R. Sivakumar, "Initial coefficient bounds for a subclass of $m$-fold symmetric biunivalent functions," Tbilisi Mathematical Journal, vol. 7, no. 2, pp. 1-10, 2014.

[6] M. Lewin, "On a coefficient problem for bi-univalent functions," Proceedings of the American Mathematical Society, vol. 18, pp. 63-68, 1967.

[7] D. A. Brannan and J. Clunie, "Aspects of contemporary complex analysis," in Proceedings of the NATO Advanced Study Institute held in Durham, England, August 26-September 6, 1974, Academic Press, New York, NY, USA, 1979.

[8] E. Netanyahu, "The minimal distance of the image boundary from the origin and the second coefficient of a univalent function in $|\mathbf{z}|<1$," Archive for Rational Mechanics and Analysis, vol. 32, pp. 100-112, 1969.

[9] D. A. Brannan and T. S. Taha, "On some classes of bi-univalent functions," Studia Universitatis Babeş-Bolyai, Mathematica, vol. 31, no. 2, pp. 70-77, 1986.

[10] S. Altınkaya and S. Yalçın, "Initial coefficient bounds for a general class of biunivalent functions," International Journal of Analysis, vol. 2014, Article ID 867871, 4 pages, 2014.

[11] Ş. Altınkaya and S. Yalçın, "Coefficient estimates for two new subclasses of biunivalent functions with respect to symmetric points," Journal of Function Spaces, vol. 2015, Article ID 145242, 5 pages, 2015.

[12] B. A. Frasin and M. K. Aouf, "New subclasses of bi-univalent functions," Applied Mathematics Letters, vol. 24, no. 9, pp. 15691573, 2011.

[13] N. Magesh and J. Yamini, "Coefficient bounds for a certain subclass of bi-univalent functions," International Mathematical Forum, vol. 8, no. 27, pp. 1337-1344, 2013.

[14] H. M. Srivastava, S. Bulut, M. Çağlar, and N. Yağmur, "Coefficient estimates for a general subclass of analytic and biunivalent functions," Filomat, vol. 27, no. 5, pp. 831-842, 2013.

[15] Q.-H. Xu, Y.-C. Gui, and H. M. Srivastava, "Coefficient estimates for a certain subclass of analytic and bi-univalent functions," Applied Mathematics Letters, vol. 25, no. 6, pp. 990-994, 2012. 
[16] S. Bulut, "Faber polynomial coefficient estimates for a comprehensive subclass of analytic bi-univalent functions," Comptes Rendus Mathematique, vol. 352, no. 6, pp. 479-484, 2014.

[17] S. G. Hamidi and J. M. Jahangiri, "Faber polynomial coefficient estimates for analytic bi-close-to-convex functions," Comptes Rendus de l'Académie des Sciences. Series I, vol. 352, no. 1, pp. 17-20, 2014.

[18] J. M. Jahangiri and S. G. Hamidi, "Coefficient estimates for certain classes of bi-univalent functions," International Journal of Mathematics and Mathematical Sciences, vol. 2013, Article ID 190560, 4 pages, 2013.

[19] G. Murugusundaramoorty, N. Magesh, and V. Prameela, "Coefficient bounds for certain subclasses of bi-univalent function," Abstract and Applied Analysis, vol. 2013, Article ID 573017, 3 pages, 2013. 


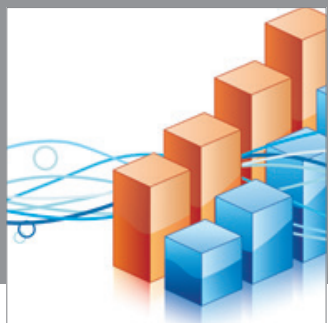

Advances in

Operations Research

mansans

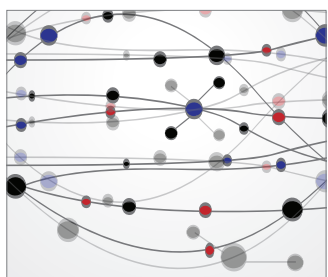

The Scientific World Journal
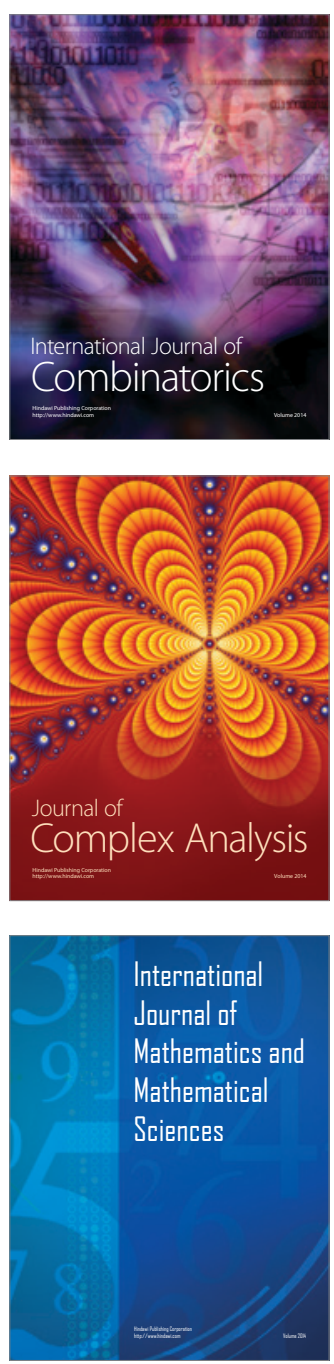
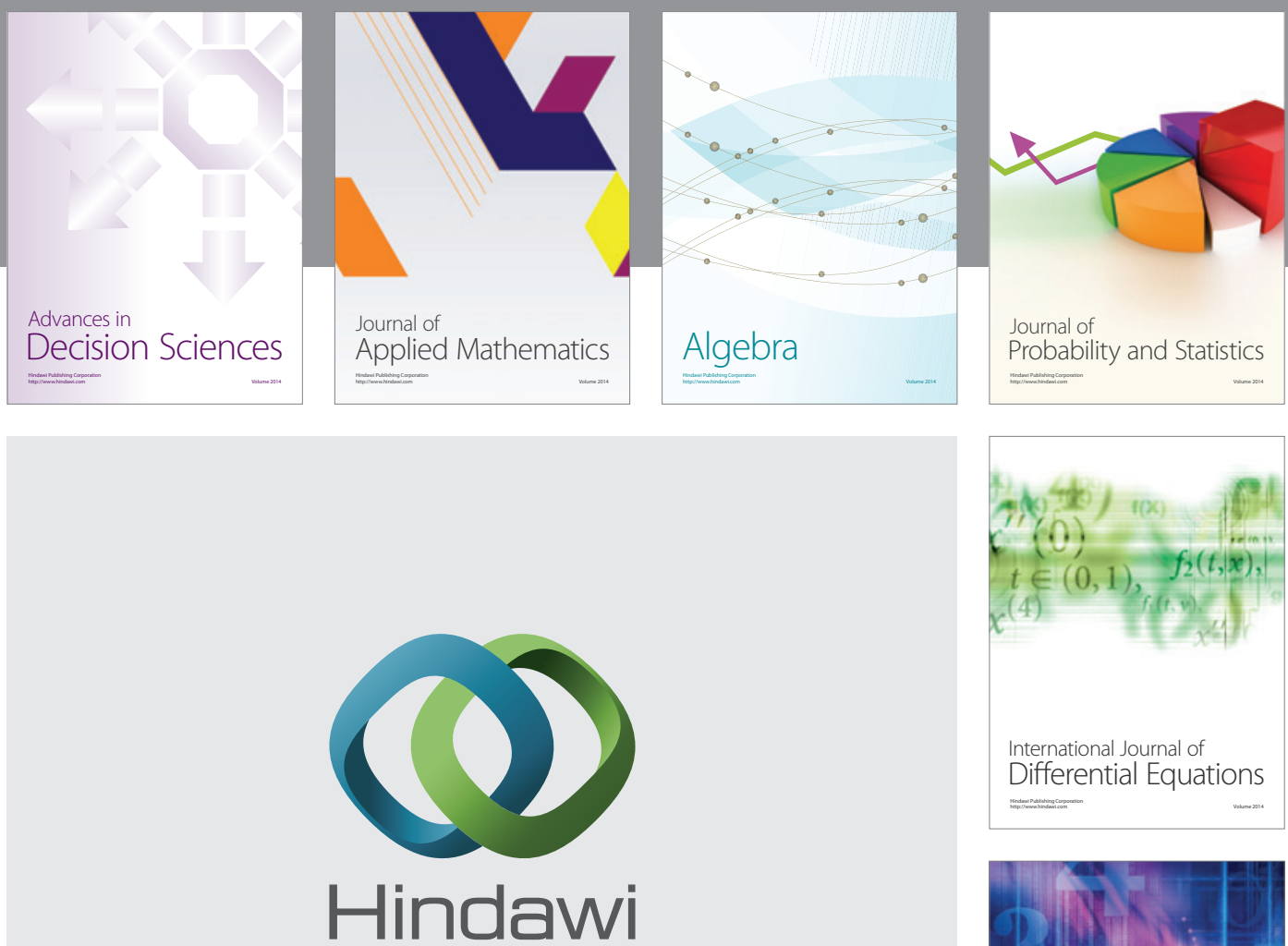

Submit your manuscripts at http://www.hindawi.com
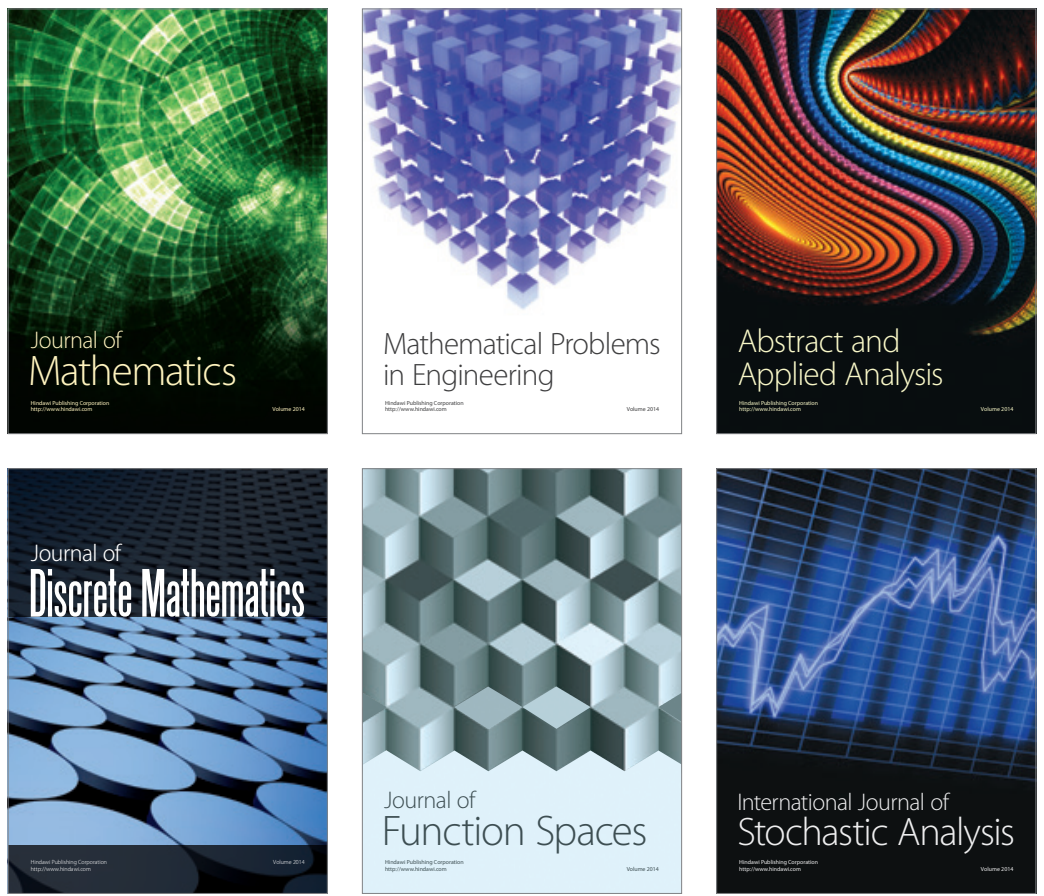

Journal of

Function Spaces

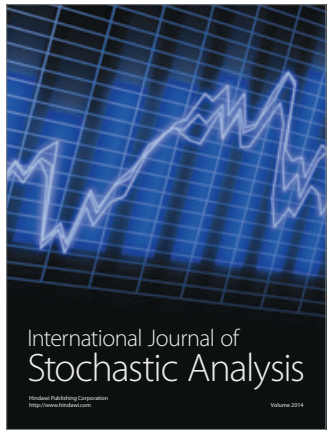

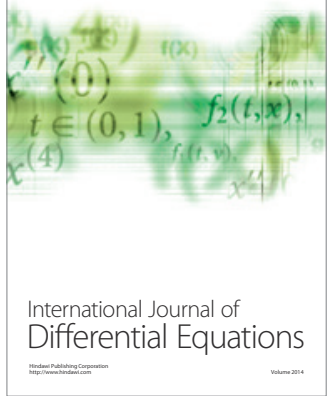
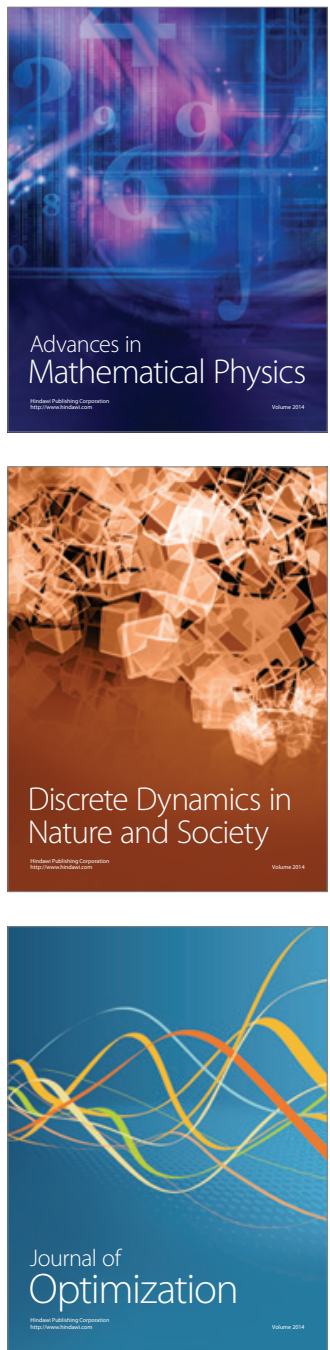